Vikramman Vignaraja

Maidstone \& Kent NHS Trust, UK

EMERGENCY

Vikramman.vignaraja@nhs.net

\title{
SIMULATION TRAINING IN PAEDIATRICS
}

cite as: Vignaraja, V. Introducing Emergency Simulation Training in Paediatrics. The Physician 2020 vol6; issue2: abp11 DOI: $10.38192 / 1 \cdot 6.2 .18$

Article Information

Epub: 27.11.2019

Published 21.08.2020

Poster presented at BAPIO National Conference, London Nov 2019

Peer reviewed by Subarna Chakravorty \& Sunil Daga

Keywords:

paediatric emergencies, simulation based training

\section{Aim}

To introduce regular paediatric emergency simulation sessions for paediatric trainees at QEH, Woolwich and to evaluate early attitudes toward its role in training.

\section{Background}

Direct repeated experience is necessary for trainees to gain confidence and competence in management of time critical, life threatening scenarios. The relative infrequency of paediatric arrest and peri-arrest situations means opportunity to gain vital experience can be limited. Simulation offers an effective educational tool to bridge the gap between theoretical knowledge and real life implementation. Multiple studies have shown that simulation in Paediatrics correlate with better health outcomes for patients ranging from an improvement in cardiopulmonary arrest survival rates, earlier identification of a sick child and reduced serious safety incidents in A\&E.

\section{Method}

We started off by surveying junior doctor attitudes towards simulation in training. My sample population included junior paediatric SHOs, GP trainees and ED SHOs. Though my sample size was small it showed strong support for paediatric simulation. We conducted the sessions in the paediatric resuscitation bay in A\&E to make the situation as realistic as possible. This has the added benefit of making sure when in a real emergency, trainees know where to find necessary equipment. Scenarios have been run by experienced registrars for small groups of trainees. Scenarios have been run by experienced registrars for small groups of trainees.
Results

We initially surveyed 10 SHO grade doctors and of these, 7 reported that they felt "very unconfident" in managing paediatric emergencies. Evaluation forms from our simulation session on managing life threatening asthma showed that 7 out of 8 doctors who attended said that they felt more confident to manage the situation after having had simulation in it.

Conclusions

These results show the effectiveness of simulation on trainee confidence in managing emergencies by experiential learning. Good facilitators are crucial to run the simulation. Without reflection, the learning process is greatly diminished.

Conflict of Interests/Comments: Nothing to declare. 